\title{
AN AVERAGING FORMULA FOR REIDEMEISTER TRACES
}

\author{
JiYOU LiU — XUEZHI ZHAO
}

\begin{abstract}
In this note, we shall give an averaging formula for Reidemeister traces, which is a simple relation among Reidemeister traces of a selfmap and those of its liftings with respect to a finite-fold regular covering.
\end{abstract}

\section{Introduction}

Let $f: X \rightarrow X$ be a self-map on a connected compact polyhedron. Assume that $f$ admits a lifting with respect to an $n$-fold regular covering $q: \bar{X} \rightarrow X$. It is well-known (cf. [2, Chapter II, 5.4 Theorem]) that there is an averaging formula

$$
L(f)=\frac{1}{n} \sum_{\bar{f} \in \operatorname{lift}(f, q)} L(\bar{f})
$$

for Lefschetz numbers. Here, $\operatorname{lift}(f, q)$ stands for the set of all liftings of $f$ with respect to the covering $q: \bar{X} \rightarrow X$. Moreover, an averaging formula

$$
N(f)=\frac{1}{n} \sum_{\bar{f} \in \operatorname{lift}(f, q)} N(\bar{f})
$$

for Nielsen numbers was obtained in [4] under some assumptions on the given self-map $f$ or the space $X$. In this note, we shall show that there does exist

2010 Mathematics Subject Classification. 55M20.

Key words and phrases. Averaging formula, Reidemeister trace, self-map, lifting, regular covering.

This work is supported by the NSF of China (11431009) and a project of Beijing Municipal Education Commission (KZ201310028030). 
an averaging formula for Reidemeister traces. The Reidemeister trace of a selfmap is also a classical invariant containing the information of both the Lefschetz number and the Nielsen number.

Our approach follows the idea in [4] and [3]. We study the relations between invariants in Nielsen fixed point theory of a self-map and those of its liftings with respect to a finite-fold covering. We focus on the deck transformation group other than fundamental group. The action of deck transformation group on the set of lifting conjugacy classes are also addressed.

Throughout this note, we always assume that

(1) $X$ is a connected compact polyhedron,

(2) $p: \widetilde{X} \rightarrow X$ is the universal covering with deck transformation group $D(\widetilde{X})$

(3) $q: \bar{X} \rightarrow X$ is an $n$-fold regular covering which is determined by a normal subgroup $H$ of $D(\widetilde{X})$, i.e. $\bar{X}=\widetilde{X} / H$, having deck transformation group $D(\bar{X})=D(\widetilde{X}) / H$,

(4) $f: X \rightarrow X$ is a self-map admitting a lifting with respect to the covering $q: \bar{X} \rightarrow X$.

We write $\operatorname{lift}(f, p)$ and $\operatorname{lift}(f, q)$ respectively for the sets of lifting of $f$ with respect to the coverings $p$ and $q$. See [2] or [3] for related notations.

\section{Fixed point classes between a finite-fold covering}

In this section, we shall study the relation between the fixed point classes of a self-map and those of its liftings with respect to a finite-fold regular covering.

Let us review some basic definitions and facts in Nielsen fixed point theory, see [2] for more details. For the given self-map $f: X \rightarrow X$, two liftings $\widetilde{f}_{1}$ and $\widetilde{f}_{2}$ of $f$ in the set lift $(f, p)$ are said to be conjugate if there is an element $\gamma \in D(\widetilde{X})$ such that $\widetilde{f}_{2}=\gamma \widetilde{f}_{1} \gamma^{-1}$. We write $[\widetilde{f}]_{f}$ for the lifting conjugacy class of $f$ containing the lifting $\widetilde{f}$. The set of all lifting conjugacy classes of $f$ is written as $\operatorname{FPC}(f)$, which was called fixed point classes data of $f$ (see [2, Chapter III, 1.10]). If $\widetilde{f}_{1}$ and $\widetilde{f}_{2}$ are conjugate, then $p\left(\operatorname{Fix} \widetilde{f}_{1}\right)=p\left(\operatorname{Fix} \widetilde{f}_{2}\right)$. For a given lifting $\tilde{f}$ of $f$, the subset $p(\operatorname{Fix} \widetilde{f})$ of fixed point set of $f$ is said to be the fixed point class of $f$ determined by the lifting conjugacy class $[\widetilde{f}]$ (or say by the lifting $\widetilde{f}$ ) if such a subset is non-empty. Here, we do distinguish lifting conjugacy classes and fixed point classes. A fixed point class of a self-map is always assumed to be a non-empty subset of the fixed point set of the given self-map.

If we choose a lifting $\tilde{f}$ as a reference, then any lifting of $f$ can be written uniquely as $\gamma \widetilde{f}$ for some $\gamma \in D(\widetilde{X})$. Hence, $\widetilde{f}$ induces an endomorphism $\widetilde{f}_{D}: D(\widetilde{X}) \rightarrow D(\widetilde{X})$ which is defined by $\widetilde{f} \gamma=\widetilde{f}_{D}(\gamma) \widetilde{f}$. By definition of $\widetilde{f}_{D}$, we know that $\gamma_{1} \tilde{f}$ and $\gamma_{2} \tilde{f}$ are conjugate, i.e. $\gamma_{2} \widetilde{f}=\gamma \gamma_{1} \tilde{f} \gamma^{-1}$, if and only 
if $\gamma_{2}=\gamma \gamma_{1} \widetilde{f}_{D}\left(\gamma^{-1}\right)$. We say that two elements $\gamma_{1}$ and $\gamma_{2}$ in $D(\tilde{X})$ are $\widetilde{f}_{D^{-}}$ conjugate if $\gamma_{2}=\gamma \gamma_{1} \tilde{f}_{D}\left(\gamma^{-1}\right)$ for some $\gamma \in D(\widetilde{X})$. Write $D(\widetilde{X})_{\widetilde{f}_{D}}$ for the set of all $\widetilde{f}_{D}$-conjugacy classes. Thus, there is a well-defined one-to-one correspondence

$$
\rho_{\widetilde{f}}: D(\widetilde{X})_{\widetilde{f}_{D}} \rightarrow \operatorname{FPC}(f), \quad \rho_{\widetilde{f}}\left([\alpha]_{\widetilde{f}_{D}}\right)=[\alpha \widetilde{f}]_{f} .
$$

Let $\bar{f} \in \operatorname{lift}(f, q)$. Then we have the following commutative diagram:

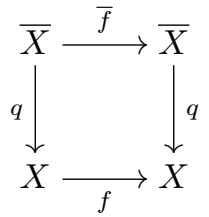

Since $X$ and $\bar{X}$ share the same universal covering space $\widetilde{X}$, any lifting of $\bar{f}$ is also a lifting of $f$. (But, the converse is not true.) By [2, Chapter III, 1.4], we have

Proposition 2.1. If the given self-map $f$ admits a lifting with respect to the finite-fold covering $q: \bar{X} \rightarrow X$, then the formula $q_{\mathrm{FPC}}\left([\tilde{f}]_{\bar{f}}\right)=[\tilde{f}]_{f}$ gives a natural map $q_{\mathrm{FPC}}: \operatorname{FPC}(\bar{f}) \rightarrow \operatorname{FPC}(f)$.

Let us consider the union of the sets of lifting conjugacy classes of all selfmaps in $\operatorname{lift}(f, q)$.

Proposition 2.2. If the given self-map $f$ admits a lifting with respect to the finite-fold covering $q: \bar{X} \rightarrow X$, then the map

$$
q_{\mathrm{FPC}}^{\sqcup}: \bigsqcup_{\bar{f} \in \operatorname{lift}(f, q)} \operatorname{FPC}(\bar{f}) \rightarrow \operatorname{FPC}(f)
$$

is surjective.

Proof. Given a lifting $\widetilde{f}$ in the set $\operatorname{lift}(f, p)$. Let $H$ be the subgroup of $D(\widetilde{X})$ corresponding to the covering $q$, i.e. $\bar{X}=\widetilde{X} / H$. For any $\alpha \in H$ and any point $\widetilde{x}_{0} \in \widetilde{X}$, we have $\widetilde{f}\left(\alpha \widetilde{x}_{0}\right)=\widetilde{f}_{D}(\alpha) \widetilde{f}\left(\widetilde{x}_{0}\right)$. Since $f$ admits a lifting with respect to the covering $q: \bar{X} \rightarrow X$, we know that $\widetilde{f}_{D}(\alpha) \in H$. It follows that $\tilde{f}$ induces a self-map $\bar{f}$ on $\bar{X}$, which is obviously a lifting of $f$ with respect to $q: \bar{X} \rightarrow X$. Note that $\widetilde{f}$ is also a lifting of $\bar{f}$ with respect to the universal covering $\bar{p}: \widetilde{X} \rightarrow \bar{X}$. We obtain that $q_{\mathrm{FPC}}^{\sqcup}\left([\widetilde{f}]_{\bar{f}}\right)=[\widetilde{f}]_{f}$.

From the proof of this proposition, we know that a lifting of $f$ with respect to the universal covering induces a unique self-map on $\bar{X}$. In other words, a lifting of $f$ is a lifting of a unique self-map in lift $(f, q)$. Thus, the union $\bigsqcup_{\bar{f} \in \operatorname{lift}(f, q)} \operatorname{FPC}(\bar{f})$ is really a disjoint union, i.e. any two elements, regarded as two subsets of all self-maps on $\widetilde{X}$, in $\bigsqcup_{\bar{f} \in \operatorname{lift}(f, q)} \operatorname{FPC}(\bar{f})$ have no intersection. 
Recall that the Reidemeister number of a self-map is defined to be the number of lifting conjugacy classes. Thus, we have

Corollary 2.3. $R(f)=\infty$ if and only if $R(\bar{f})=\infty$ for some lifting $\bar{f} \in$ $\operatorname{lift}(f, q)$.

Definition 2.4. Let $\bar{f} \in \operatorname{lift}(f, q)$. A fixed point class $F$ of $f$ is said to be covered by a fixed point class $\bar{F}$ of $\bar{f}$ if $\bar{F}$ and $F$ are determined by the same lifting.

Next lemma gives some geometric meaning of above definition and the map $q_{\mathrm{FPC}}^{\sqcup}$

Lemma 2.5. Let $\bar{f} \in \operatorname{lift}(f, q)$, and let $\bar{F}$ and $F$ be respectively fixed point classes of $\bar{f}$ and $f$. Then the following statements are equivalent:

(a) $F$ is covered by $\bar{F}$,

(b) $q(\bar{F})=F$,

(c) $q(\bar{F}) \cap F \neq \emptyset$.

Proof. Note that $X$ and its finite-fold covering space $\bar{X}$ share the same universal covering space $\widetilde{X}$. Write $\bar{p}: \widetilde{X} \rightarrow \bar{X}$ for the universal covering of $\bar{X}$.

From (a) to (b). Since $F$ is covered by $\bar{F}$, by definition, there is a lifting $\tilde{f}: \widetilde{X} \rightarrow \widetilde{X}$ of both $\bar{f}$ and $f$ such that $\bar{F}=\bar{p}(\operatorname{Fix} \tilde{f})$ and $F=p(\operatorname{Fix} \widetilde{f})$. Note that $q \circ \bar{p}=p: \tilde{X} \rightarrow X$. We obtain (b).

From (b) to (c). This is trivial, because $\bar{F}$ and $F$ are both non-empty sets.

From (c) to (a). Let $x_{0} \in q(\bar{F}) \cap F$. We can find a point $\bar{x}_{0} \in \bar{F} \cap q^{-1}\left(x_{0}\right)$. Let $\widetilde{f}$ be a lifting of $\bar{f}$ determining $\bar{F}$. Thus, there is a point $\widetilde{x}_{0} \in \bar{p}^{-1}\left(\bar{x}_{0}\right)$ such that $\tilde{f}\left(\widetilde{x}_{0}\right)=\widetilde{x}_{0}$. Note that $\widetilde{x}_{0} \in p^{-1}\left(x_{0}\right)$, and that $f\left(x_{0}\right)=x_{0}$. We have that $x_{0} \in p(\operatorname{Fix} \tilde{f})$. It follows that $F=p(\operatorname{Fix} \widetilde{f})$, i.e. $F$ is also determined by $\widetilde{f}$.

Now, we illustrate an example. Let $X$ be the unite circle $S^{1}$. Then its universal covering map $p: \widetilde{X}=\mathbb{R} \rightarrow X$ is given by $p(t)=e^{2 \pi t i}$. The deck transformation group $D(\tilde{X}) \cong \mathbb{Z}$ is generated by $\gamma$, where $\gamma(t)=t+1$.

A self-map $f: X \rightarrow X$ is defined by $f\left(e^{\theta i}\right)=e^{4 \theta i}$. There is a lifting $\tilde{f}$ given by $\tilde{f}(t)=4 t$. Then $\operatorname{lift}(f, p)=\left\{\gamma^{k} \widetilde{f} \mid k \in \mathbb{Z}\right\}$. Since $t \stackrel{\gamma^{-1}}{\longmapsto} t-1 \stackrel{\widetilde{f}}{\longmapsto} 4 t-4 \stackrel{\gamma}{\longmapsto}$ $4 t-3$, i.e. $\gamma \widetilde{f} \gamma^{-1}=\gamma^{-3} \widetilde{f}$, we obtain that $\operatorname{FPC}(f)=\left\{[\tilde{f}]_{f},[\gamma \tilde{f}]_{f},\left[\gamma^{2} \widetilde{f}\right]_{f}\right\}$, where $\left[\gamma^{j} \widetilde{f}\right]_{f}=\left\{\gamma^{j+3 l} \widetilde{f} \mid l \in \mathbb{Z}\right\}$ for $j=0,1,2$.

A two-fold covering $q: \bar{X}=S^{1} \rightarrow X$ is defined by $q\left(e^{\theta i}\right)=e^{2 \theta i}$. Thus, $\operatorname{lift}(f, q)=\{\bar{f}, \tau \bar{f}\}$, where $\bar{f}\left(e^{\theta i}\right)=e^{4 \theta i}$ and $\tau\left(e^{\theta i}\right)=e^{(\theta+\pi) i}\left(=-e^{\theta i}\right)$. Note that $q$ is determined by the subgroup $\left\{\gamma^{2 l} \mid l \in \mathbb{Z}\right\}$ of $D(\widetilde{X})$. The universal covering $\bar{p}: \widetilde{X} \rightarrow \bar{X}$ is given by $\bar{p}(t)=e^{\pi t i}$, which is also implied by the relation $p=q \circ \bar{p}$. Thus, $\operatorname{lift}(\bar{f}, \bar{p})=\left\{\gamma^{2 l} \widetilde{f} \mid l \in \mathbb{Z}\right\}$, while $\operatorname{lift}(\tau \bar{f}, \bar{p})=\left\{\gamma^{2 l+1} \tilde{f} \mid l \in \mathbb{Z}\right\}$. We obtain that $\operatorname{FPC}(\bar{f})=\left\{[\widetilde{f}]_{\bar{f}},\left[\gamma^{2} \widetilde{f}\right]_{\bar{f}},\left[\gamma^{4} \widetilde{f}\right]_{\bar{f}}\right\}$, where $\left[\gamma^{j} \widetilde{f}\right]_{\bar{f}}=\left\{\gamma^{j+6 l} \widetilde{f} \mid l \in \mathbb{Z}\right\}$ 
for $j=0,2,4$. We also obtain that $\operatorname{FPC}(\tau \bar{f})=\left\{[\gamma \widetilde{f}]_{\tau \bar{f}},\left[\gamma^{3} \widetilde{f}\right]_{\tau \bar{f}},\left[\gamma^{5} \widetilde{f}\right]_{\tau \bar{f}}\right\}$, where $\left[\gamma^{j} \widetilde{f}\right]_{\tau \bar{f}}=\left\{\gamma^{j+6 l} \tilde{f} \mid l \in \mathbb{Z}\right\}$ for $j=1,3,5$.

It is obvious that

$$
[\widetilde{f}]_{f}=[\widetilde{f}]_{\bar{f}} \sqcup\left[\gamma^{3} \widetilde{f}\right]_{\tau \bar{f}}, \quad[\gamma \widetilde{f}]_{f}=\left[\gamma^{4} \widetilde{f}\right]_{\bar{f}} \sqcup[\gamma \widetilde{f}]_{\tau \bar{f}}, \quad\left[\gamma^{2} \widetilde{f}\right]_{f}=\left[\gamma^{2} \widetilde{f}\right]_{\bar{f}} \sqcup\left[\gamma^{5} \widetilde{f}\right]_{\tau \bar{f}} .
$$

Thus, we have

$$
\begin{aligned}
\left(q_{\mathrm{FPC}}^{\sqcup}\right)^{-1}\left([\widetilde{f}]_{f}\right) & =\left\{[\widetilde{f}]_{\bar{f}},\left[\gamma^{3} \widetilde{f}\right]_{\tau \bar{f}}\right\}, \\
\left(q_{\mathrm{FPC}}^{\sqcup}\right)^{-1}\left([\gamma \widetilde{f}]_{f}\right)^{-1} & =\left\{\left[\gamma^{4} \widetilde{f}\right]_{\bar{f}},[\gamma \widetilde{f}]_{\tau \bar{f}}\right\}, \\
\left(q_{\mathrm{FPC}}^{\sqcup}\right)^{-1}\left(\left[\gamma^{2} \widetilde{f}\right]_{f}\right)^{-1} & =\left\{\left[\gamma^{2} \widetilde{f}\right]_{f},\left[\gamma^{5} \widetilde{f}\right]_{\tau \bar{f}}\right\} .
\end{aligned}
$$

It is easy to check that

$$
\begin{array}{rlrl}
p\left(\operatorname{Fix} \gamma^{l} \widetilde{f}\right) & =\left\{e^{-2 \pi l i / 3}\right\}, & l=0,1,2 ; \\
\bar{p}\left(\operatorname{Fix} \gamma^{m} \widetilde{f}\right) & =\left\{e^{-\pi m i / 3}\right\}, & & m=0, \ldots, 5 .
\end{array}
$$

Thus, each lifting conjugacy class determines a fixed point class. Each fixed point class of $f$ is covered by two fixed point classes: one is that of $\bar{f}$, the other is that of $\tau \bar{f}$. Clearly, the fixed point class $\left\{e^{0}\right\}$ is covered by $\left\{e^{0}\right\}$ and $\left\{e^{-\pi i}\right\}$, $\left\{e^{-2 \pi i / 3}\right\}$ is covered by $\left\{e^{-\pi i / 3}\right\}$ and $\left\{e^{-4 \pi i / 3}\right\}$, and $\left\{e^{-4 \pi i / 3}\right\}$ is covered by $\left\{e^{-2 \pi i / 3}\right\}$ and $\left\{e^{-5 \pi i / 3}\right\}$. Moreover, $q^{-1}\left(\left\{e^{0}\right\}\right)=\left\{e^{0}, e^{-\pi i}\right\}, q^{-1}\left(\left\{e^{-2 \pi i / 3}\right\}\right)=$ $\left\{e^{-\pi i / 3}, e^{-4 \pi i / 3}\right\}$, and $q^{-1}\left(\left\{e^{-4 \pi i / 3}\right\}\right)=\left\{e^{-2 \pi i / 3}, e^{-5 \pi i / 3}\right\}$.

We would like to mention that the number of the fixed point classes covering a given fixed point class maybe smaller than the fold number of $q$ (see the example in the final section of this note). A general result will be given in Lemma 3.3.

Finally, we make some remarks about the relations of our definition and existed invariants. Let $F$ be a fixed point class of $f$ determined by a lifting $\widetilde{f}$. There are two subgroups $q_{D}\left(\operatorname{Fix} \widetilde{f}_{D}\right)$ and Fix $\widetilde{\widetilde{f}_{D}}$ of $D(\bar{X})$. Here, $q_{D}: D(\widetilde{X}) \rightarrow$ $D(\bar{X})=D(\widetilde{X}) / H$ is the quotient homomorphism, and $\widetilde{\widetilde{f}_{D}}: D(\bar{X}) \rightarrow D(\bar{X})$ is the endomorphism induced by $\widetilde{f}_{D}: D(\widetilde{X}) \rightarrow D(\widetilde{X})$. Their orders are written as $J_{F}$ and $I_{F}$, respectively (see [3, Corollary 3.11]). Since $q_{D}\left(\right.$ Fix $\left.\widetilde{f}_{D}\right)$ is a subgroup of Fix $\widetilde{\widetilde{f}_{D}}, J_{F}$ is a factor of $I_{F}$. The two numbers are both independent of the choice of liftings, and totally determined by the fixed point class $F$ itself. They count the fold numbers between the given fixed point class $F$ and those covering it. More precisely,

Proposition 2.6 (cf. [3, Lemma 3.4]). Let $F$ be a fixed point class of $f$, admitting a lifting with respect to a finite-fold regular covering $q: \bar{X} \rightarrow X$. Then

(a) $q \mid: \bar{F} \rightarrow F$ is a $J_{F}$-fold covering for any fixed point class $\bar{F}$ covering $F$;

(b) $q \mid: \operatorname{Fix} \bar{f} \cap q^{-1}(F) \rightarrow F$ is an $I_{F}$-fold covering for any $\bar{f} \in \operatorname{lift}(f, q)$ with Fix $\bar{f} \cap q^{-1}(F) \neq \emptyset$. 


\section{Actions of deck transformation groups}

In this section, we shall discuss the actions of deck transformation groups on lifting conjugacy classes.

THEOREM 3.1. Let $f: X \rightarrow X$ be a self-map, admitting a lifting with respect to a finite-fold regular covering $q: \bar{X} \rightarrow X$ which is determined by a normal subgroup $H$ of $D(\widetilde{X})$. Define a natural action of $D(\widetilde{X})$ on $\bigsqcup_{\bar{f} \in \operatorname{lift}(f, q)} \operatorname{FPC}(\bar{f})$ by

$$
(\eta,[\widetilde{f}]) \mapsto\left[\eta \widetilde{f} \eta^{-1}\right]
$$

Then

(a) such an action induces an action of $D(\bar{X})=D(\tilde{X}) / H$ on $\bigsqcup_{\bar{f} \in \operatorname{lift}(f, q)} \operatorname{FPC}(\bar{f})$;

(b) the isotropy group of the $D(\bar{X})$-action at the lifting conjugacy class $[\widetilde{f}]$ is $q_{D}\left(\operatorname{Fix} \widetilde{f}_{D}\right)$, where $q_{D}: D(\widetilde{X}) \rightarrow D(\bar{X})=D(\tilde{X}) / H$ is the quotient homomorphism;

(c) two elements in $\bigsqcup_{\bar{f} \in \operatorname{lift}(f, q)} \operatorname{FPC}(\bar{f})$ lie in the same $D(\bar{X})$-action orbit if and only if they have the same image of

$$
q_{\mathrm{FPC}}^{\sqcup}: \bigsqcup_{\bar{f} \in \operatorname{lift}(f, q)} \operatorname{FPC}(\bar{f}) \rightarrow \operatorname{FPC}(f) .
$$

Proof. Let $\eta$ be an element in $D(\tilde{X})$. If $\left[\eta \widetilde{f} \eta^{-1}\right]$ and $[\widetilde{f}]$ are the same lifting conjugacy class of some $\bar{f} \in \operatorname{lift}(f, q)$, then there is an element $\alpha \in H$ such that $\eta \widetilde{f} \eta^{-1}=\alpha \widetilde{f} \alpha^{-1}$. This is equivalent to say that $\eta \widetilde{f}_{D}\left(\eta^{-1}\right)=\alpha \widetilde{f}_{D}\left(\alpha^{-1}\right)$, i.e. $\eta^{-1} \alpha \in \operatorname{Fix} \widetilde{f}_{D}$. Thus, isotropy group of $D(\widetilde{X})$-action at the lifting conjugacy class $[\widetilde{f}]$ is $H\left(\operatorname{Fix} \widetilde{f}_{D}\right)=\left\{\alpha \beta \mid \alpha \in H, \beta \in \operatorname{Fix} \widetilde{f}_{D}\right\}$. It is really a subgroup of $D(\tilde{X})$ because $H$ is a normal subgroup of $D(\widetilde{X})$. Since $H\left(\operatorname{Fix} \widetilde{f}_{D}\right)$ contains $H$ as a subgroup for any lifting $\tilde{f}, H$ acts trivially on $\bigsqcup_{\bar{f} \in \operatorname{lift}(f, q)} \operatorname{FPC}(\bar{f})$. Hence, there must be an induced $D(\bar{X})$-action. We proved (a).

Project the isotropy group of $D(\tilde{X})$-action by $q_{D}: D(\tilde{X}) \rightarrow D(\bar{X})$. We know that the isotropy group of $D(\bar{X})$-action at $[\widetilde{f}]$ is $q_{D}\left(H\left(\operatorname{Fix} \widetilde{f}_{D}\right)\right)=q_{D}\left(\operatorname{Fix} \widetilde{f}_{D}\right)$. Thus, we obtain (b).

The conclusion (c) is obvious by definition of $D(\bar{X})$-action and $q_{\mathrm{FPC}}^{\sqcup}$.

Corollary 3.2. The set $\operatorname{FPC}(f)$ is just the orbit set $\bigsqcup_{\bar{f} \in \operatorname{lift}(f, q)} \operatorname{FPC}(\bar{f}) / D(\tilde{X})$, or $\bigsqcup_{\bar{f} \in \operatorname{lift}(f, q)} \operatorname{FPC}(\bar{f}) / D(\bar{X})$, and hence $q_{\mathrm{FPC}}^{\sqcup}$ can be regarded as a quotient map.

It should be mentioned that the $D(\bar{X})$-action on the union of all fixed point classes of all self-maps in $\operatorname{lift}(f, q)$ is exactly its usual action (being considered as homeomorphisms) on $\bar{X}$. 
Lemma 3.3. Let $q: \bar{X} \rightarrow X$ be an $n$-fold regular covering. Let $F$ be a fixed point class of $f: X \rightarrow X$, where $f$ admits a lifting with respect to $q$. Then the number of the fixed point classes of self-maps in $\operatorname{lift}(f, q)$ covering $F$ is $n / J_{F}$.

Proof. Let $F$ be a fixed point class of $f$ determined by a lifting $\widetilde{f}$, i.e. $p(\operatorname{Fix} \widetilde{f})=F$, which is a non-empty subset of $\widetilde{X}$. For any $\left[\widetilde{f}^{\prime}\right] \in\left(q_{\mathrm{FPC}}^{\sqcup}\right)^{-1}\left([\widetilde{f}]_{f}\right)$, we have $\widetilde{f}^{\prime}=\gamma \widetilde{f} \gamma^{-1}$ for some $\gamma \in D(\widetilde{X})$ by (c) of Theorem 3.1. Note that $\operatorname{Fix} \gamma \widetilde{f} \gamma^{-1}=\gamma(\operatorname{Fix} \widetilde{f}) \neq \emptyset$. The lifting conjugacy class $\left[\widetilde{f}^{\prime}\right]$ determines a fixed point class of a self-map in $\operatorname{lift}(f, q)$. Thus, the number of the fixed point classes covering $F$ is equal to $\sharp\left(q_{\mathrm{FPC}}^{\sqcup}\right)^{-1}\left([\widetilde{f}]_{f}\right)$, which is just $\sharp D(\bar{X}) / \sharp q_{D}\left(\operatorname{Fix} \widetilde{f}_{D}\right)=n / J_{F}$ by (b) and (c) of Theorem 3.1.

Proposition 3.4. Let $f: X \rightarrow X$ be a self-map admitting a lifting with respect to an $n$-fold regular covering $q: \bar{X} \rightarrow X$. Then

$$
\sum_{F \in \mathcal{N}(f)} \frac{n}{J_{F}}=\sum_{\bar{f} \in \operatorname{lift}(f, q)} N(\bar{f})
$$

where $\mathcal{N}(f)$ is the set of all essential fixed point classes of $f$.

Proof. By [4, Lemma 2.5] or [3, Lemma 3.4], if a fixed point class $\bar{F}$ covers a fixed point class $F$ of $f$, then $\bar{F}$ and $F$ has the same essentiality. From Proposition 2.2, we know that each essential fixed point class of some self-map in lift $(f, q)$ cover an essential fixed point class of $f$. It follows that

$$
\sum_{\bar{f} \in \operatorname{lift}(f, q)} N(\bar{f})=\sum_{F \in \mathcal{N}(f)} \sharp\{\text { fixed point classes covering } F\}=\sum_{F \in \mathcal{N}(f)} \frac{n}{J_{F}},
$$

by Lemma 3.3 .

Since $1 \leq J_{F} \leq n=\sharp D(\bar{X})$, we have

Corollary 3.5 .

$$
\frac{1}{n} \sum_{\bar{f} \in \operatorname{lift}(f, q)} N(\bar{f}) \leq N(f) \leq \sum_{\bar{f} \in \operatorname{lift}(f, q)} N(\bar{f}) .
$$

By results above, we have the following:

Proposition 3.6. Let $q: \bar{X} \rightarrow X$ be an $n$-fold regular covering. A self-map $f: X \rightarrow X$ admits a lifting with respect to $q$. Then the following statements are equivalentrom:

(a) $N(f)=\sum_{\bar{f} \in \operatorname{lift}(f, q)} N(\bar{f})$;

(b) $J_{F}=n$ for any essential fixed point class $F$ of $f$;

(c) $D(\bar{X})$ acts trivially at each lifting conjugacy class which determines an essential fixed point class of some self-map in $\operatorname{lift}(f, q)$; 
(d) each essential fixed point class of $f$ is covered by unique fixed point class of self-maps in $\operatorname{lift}(f, q)$, i.e. $\sharp\left(q_{\mathrm{FPC}}^{\sqcup}\right)^{-1}\left([\widetilde{f}]_{f}\right)=1$ if the lifting conjugacy class $[\widetilde{f}]_{f}$ determines an essential fixed point class of $f$;

(e) $\operatorname{ind}(\bar{f}, \bar{F})=n \operatorname{ind}(f, F)$ for any essential fixed point class $F$ of $f$ and any fixed point class $\bar{F}$ of any self-map in $\operatorname{lift}(f, q)$ covering $F$.

Proof. By Proposition 3.4,

$$
\sum_{F \in \mathcal{N}(f)} \frac{n}{J_{F}}=\sum_{\bar{f} \in \operatorname{lift}(f, q)} N(\bar{f}) .
$$

The number of summands in left side of this equality is $N(f)$, which is the number of essential fixed point classes of $f$. Hence, (a) holds if and only if $n / J_{F}=1$, i.e. $n=J_{F}$ for all essential fixed point class of $f$. We obtain the equivalence between (a) and (b).

The Definition 2.4 and the item (b) and (c) of Theorem 3.1 imply that (b) and (c) are equivalent. The equivalence between (b) and (d) is given by Lemma 3.3. By the homotopy invariance of indices of fixed point classes, we may assume that each fixed point class is a finite set. Thus, equivalence between (d) and (e) follows directly from the definition of fixed point index.

Similarly, we can prove

Proposition 3.7 (cf. [4, Theorem 3.1]). Let $q: \bar{X} \rightarrow X$ be an $n$-fold regular covering. A self-map $f: X \rightarrow X$ admits a lifting with respect to $q$. Then the following statements are equivalent:

(a) $N(f)=(1 / n) \sum_{\bar{f} \in \operatorname{lift}(f, q)} N(\bar{f})$;

(b) $J_{F}=1$ for any essential fixed point class $F$ of $f$;

(c) $D(\bar{X})$ acts freely on lifting conjugacy classes which determine essential fixed point classes of self-maps in $\operatorname{lift}(f, q)$;

(d) each essential fixed point class of $f$ is covered by $n$ fixed point classes of self-maps in $\operatorname{lift}(f, q)$, i.e. $\sharp\left(q_{\mathrm{FPC}}^{\sqcup}\right)^{-1}\left([\tilde{f}]_{f}\right)=n$ if the lifting conjugacy class $[\widetilde{f}]_{f}$ determines an essential fixed point class of $f$;

(e) $\operatorname{ind}(\bar{f}, \bar{F})=\operatorname{ind}(f, F)$ for any essential fixed point class $F$ of $f$ and any fixed point class $\bar{F}$ of any self-map in $\operatorname{lift}(f, q)$ covering $F$.

\section{An averaging formula}

The so-called Reidemaister trace of a self-map was introduced by Reidemaister in [5]. In this note, we use a modernized treatment given in [1].

Choose a lifting $\widetilde{f}$ of $f$ with respect to the universal covering $p: \widetilde{X} \rightarrow X$. Recall that if $\left\{\left[\gamma_{j} \widetilde{f}\right]_{f} \mid j=1, \ldots, k\right\}$ is the set of all lifting conjugacy classes of $f$ determining essential fixed point classes, where each $\gamma_{j}$ lies in $D(\widetilde{X})$, then the 
Reidemeister trace $R T(f, \tilde{f})$ of $f$ is defined to be

$$
\sum_{j=1}^{k} \operatorname{ind}\left(f, p\left(\operatorname{Fix} \gamma_{j} \widetilde{f}\right)\right) \rho_{\widetilde{f}}^{-1}\left(\left[\gamma_{j} \widetilde{f}\right]_{f}\right)=\sum_{j=1}^{k} \operatorname{ind}\left(f, p\left(\operatorname{Fix} \gamma_{j} \widetilde{f}\right)\right)\left[\gamma_{j}\right]_{\widetilde{f}_{D}}
$$

which is an element in the free $\mathbb{Z}$-module generated by the set $D(\widetilde{X})_{\widetilde{f}_{D}}$ of $\widetilde{f}_{D^{-}}$ conjugacy classes $\left(\left[1\right.\right.$, Theorem 1.13]). Here, $\rho_{\tilde{f}}$ is the one-to-one correspondence from $D(\widetilde{X})_{\widetilde{f}_{D}}$ to the set $\operatorname{FPC}(f)$, as in $(2.1)$.

Since $\gamma_{j} \widetilde{f}=\gamma_{j} \alpha^{-1} \alpha \tilde{f}$ for any $\alpha \in D(\widetilde{X})$, we have that $\rho_{\alpha \widetilde{f}}^{-1}\left(\left[\gamma_{j} \widetilde{f}\right]_{f}\right)=$ $\left[\gamma_{j} \alpha^{-1}\right]_{(\alpha \widetilde{f})_{D}}$. It follows that

$$
R T(f, \alpha \widetilde{f})=\sum_{j=1}^{k} \operatorname{ind}\left(f, p\left(\operatorname{Fix} \gamma_{j} \widetilde{f}\right)\right)\left[\gamma_{j} \alpha^{-1}\right]_{(\alpha \widetilde{f})_{D}},
$$

meaning that the Reidemeister trace does depend on the choice of reference lifting. Nevertheless, the endomorphisms $\widetilde{f}_{D}$ and $(\alpha \widetilde{f})_{D}$ induced by different reference lifting is different generally, because $(\alpha \widetilde{f})_{D}(\gamma)=\alpha \widetilde{f}_{D}(\gamma) \alpha^{-1}$ for all $\gamma \in D(\widetilde{X})$.

THEOREM 4.1. Let $q: \bar{X} \rightarrow X$ be an $n$-fold regular covering which is determined by a normal subgroup $H$ of $D(\widetilde{X})$, and let $\beta_{j}, j=1, \ldots, n$ be elements in $D(\widetilde{X})$ representing all elements in $D(\bar{X})=D(\widetilde{X}) / H$. Then

$$
R T(f, \tilde{f})=\frac{1}{n} \sum_{j=1}^{n} \rho_{\widetilde{f}}^{-1} \circ q_{\mathrm{FPC}}^{\sqcup} \circ \rho_{\beta_{j} \widetilde{f}}\left(R T\left(\bar{f}_{\beta_{j}}, \beta_{j} \widetilde{f}\right)\right),
$$

where $\widetilde{f}$ is a chosen lifting of $f, \bar{f}_{\beta_{j}}$ is the self-map on $\bar{X}$ induced by $\beta_{j} \widetilde{f}$, and the correspondence

$$
\rho_{\widetilde{f}}^{-1} \circ q_{\mathrm{FPC}}^{\sqcup} \circ \rho_{\beta_{j} \tilde{f}}: D(\widetilde{X}, \bar{p})_{\left(\beta_{j} \widetilde{f}\right)_{D}} \rightarrow D(\widetilde{X})_{\widetilde{f}_{D}}
$$

is extended into the homomorphism of $\mathbb{Z}$-modules by identifying their coefficients.

Proof. Let $F$ be an essential fixed point class of $f$ determined by $[\gamma \widetilde{f}]_{f}$. By Lemma 3.3, there are $n / J_{F}$ fixed point classes covering $F$. By $[4$, Lemma 2.5] or [3, Lemma 3.4], each fixed point class covering $F$ has index $J_{F} \operatorname{ind}(f, F)$. Thus, the sum of indices of fixed point classes covering $F$ is $n \cdot \operatorname{ind}(f, F)$. This implies that in the summation

$$
\sum_{j=1}^{n} \rho_{\widetilde{f}}^{-1} \circ q_{\mathrm{FPC}}^{\sqcup} \circ \rho_{\beta_{j} \widetilde{f}}\left(R T\left(\bar{f}_{\beta_{j}}, \beta_{j} \tilde{f}\right)\right),
$$

the coefficient of the item $[\gamma]_{\widetilde{f}_{D}}$ is $n \cdot \operatorname{ind}(f, F)$. Hence, this summation is exactly $n R T(f, \tilde{f})$. This is what we want to prove. 
Actually, the correspondence $\rho_{\widetilde{f}}^{-1} \circ q_{\mathrm{FPC}}^{\sqcup} \circ \rho_{\beta_{j} \widetilde{f}}$ is given by

$$
\rho_{\widetilde{f}}^{-1} \circ q_{\mathrm{FPC}}^{\sqcup} \circ \rho_{\beta_{j} \widetilde{f}}\left([\gamma]_{\left(\beta_{j} \widetilde{f}\right)_{D}}\right)=\left[\gamma \beta_{j}\right]_{\widetilde{f}_{D}},
$$

for any $\gamma \in H \subseteq D(\widetilde{X})$ and any $j$ with $1 \leq j \leq n$. The set $\left\{\bar{f}_{\beta_{j}} \mid 1 \leq j \leq n\right\}$ is just the set $\operatorname{lift}(f, q)$ of all liftings of $f$ with respect to the covering $q: \bar{X} \rightarrow X$.

Finally, we shall give an example illustrating these Reidemeister traces in our averaging formula.

Let $f: R P^{2} \rightarrow R P^{2}$ be the identity map. Consider the universal covering $p: S^{2} \rightarrow R P^{2}$ with the deck transformation group $D\left(S^{2}\right)=\left\{\operatorname{id}_{S^{2}}, \alpha\right\}$, where $\alpha$ is the antipole map. Clearly, $f$ has two liftings $\tilde{f}=\operatorname{id}_{S^{2}}$ and $\tilde{f}^{\prime}=\alpha \tilde{f}$. Since $\alpha \operatorname{id}_{S^{2}} \alpha^{-1}=\operatorname{id}_{S^{2}}$, there are two lifting conjugcy classes, i.e. $\operatorname{FPC}(f)=$ $\left\{[\tilde{f}]_{f},[\alpha \widetilde{f}]_{f}\right\}$. Because $\chi\left(R P^{2}\right)=1$, we have that $\operatorname{ind}(f, p(\operatorname{Fix} \tilde{f}))=1$. Since $\alpha$ is fixed point free, the fixed point class $p(\operatorname{Fix} \alpha \widetilde{f})$ has zero index. Hence, $R T(f, \widetilde{f})=[1]_{\widetilde{f}_{D}}$.

Consider the map $q=p: S^{2} \rightarrow R P^{2}$ as a 2-fold regular covering. Then $\operatorname{lift}(f, q)=\left\{\operatorname{id}_{S^{2}}, \alpha\right\}$. Since $S^{2}$ is simply-connected, as self-maps on $S^{2}$, either $\operatorname{id}_{S^{2}}$ or $\alpha$ has a unique lifting, which are respectively $\tilde{f}=\operatorname{id}_{S^{2}}$ and $\alpha \tilde{f}=\alpha$, and hence has a unique lifting conjugacy class. Since $\chi\left(S^{2}\right)=2$, the unique fixed point class of $i d_{S^{2}}$ has index 2 . Hence, we have that $R T\left(\operatorname{id}_{S^{2}}, \widetilde{f}\right)=2[1]_{\widetilde{f}_{D}}$. We have that

$$
2[1]_{\widetilde{f}_{D}} \stackrel{\rho_{\widetilde{f}}}{\longmapsto} 2[\widetilde{f}]_{\mathrm{id}_{S^{2}}} \stackrel{q_{\mathrm{FPC}}=p_{\mathrm{FPC}}}{\longmapsto} 2[\widetilde{f}]_{f} \stackrel{\rho_{\tilde{f}}^{-1}}{\longmapsto} 2[1]_{\widetilde{f}_{D}}
$$

Since $\alpha$ has no fixed point, we have that $R T(\alpha, \alpha \widetilde{f})=0$, which is sent obviously to the zero element in $\mathbb{Z} D\left(S^{2}\right)_{\widetilde{f}_{D}}$. Thus, we verify our averaging formula for Reidemeister traces:

$$
R T(f, \widetilde{f})=[1]_{\widetilde{f}_{D}}=\frac{1}{2}\left([1]_{\widetilde{f}_{D}}+0\right)=\frac{1}{2}\left(\rho_{\widetilde{f}}^{-1} \circ q_{\mathrm{FPC}}^{\sqcup} \circ \rho_{\widetilde{f}}\left(R T\left(\operatorname{id}_{S^{2}}, \widetilde{f}\right)\right)+0\right) .
$$

By [4, Example 3.3], we know that the averaging formula does not hold for Nielsen numbers. Especially, in our example,

$$
N(f)=1 \neq \frac{1}{2}(1+0)=\frac{1}{2}\left(N\left(\operatorname{id}_{S^{2}}\right)+N(\alpha)\right) .
$$

From Proposition 3.7, the reason is that the number of fixed point classes of self-maps in $\operatorname{lift}(f, q)$ covering the unique essential fixed point class of $f$ is 1 , which is smaller than the fold number $n=2$ of $q$.

Acknowledgements. The authors like to thank the referee for his helpful comments and suggestions which greatly improve the statements in this note. 


\section{REFERENCES}

[1] S. Husseini, Generalized Lefschetz numbers, Trans. Amer. Math. Soc. 272 (1982), 247274.

[2] B. Jiang, Lectures on the Nielsen Fixed Point Theory, Contemp. Math. 14, Amer. Math. Soc., Providence 1983.

[3] J. JeZIERSki, Nielsen number of a covering map, Fixed Point Theory Appl. 2006, Special Issue, Art. ID 37807, 11 pp.

[4] S.W. Kim, J.B. Lee And K.B. Lee, Averaging formula for Nielsen numbers, Nagoya Math. J. 178 (2005), 37-53.

[5] K. Reidemeister, Automorphismen von Homotopiettenringen, Math. Ann. 112 (1936), $586-593$

Manuscript received July 26, 2012

JIYOU LIU

Beijing University of Posts and Telecommunications

Beijing 100876, P.R. CHINA

Xuezhi Zhao

Department of Mathematics

Institute of Mathematics and Interdisciplinary Science

Capital Normal University

Beijing 100048, P.R. CHINA

E-mail address: zhaoxve@mail.cnu.edu.cn 